\title{
Identification of Oxidative Degradation Products of Lansoprazole by Using High Resolution Mass Spectrometry Spectral Data
}

\author{
Dev Kant Shandilya' ${ }^{1}$, Rekha Israni ${ }^{1}$, Peter Edward Joseph ${ }^{2}$, Anoop Kumar ${ }^{3}$, Manoj Pillai ${ }^{3}$ \\ ${ }^{1}$ Department of Research, Bhagwant University, Ajmer, India \\ ${ }^{2}$ Department of Chemistry, ST. Johns College, Agra, India \\ ${ }^{3}$ Sciex, Gurgaon, India \\ Email: devkantshandilya@hotmail.com
}

How to cite this paper: Shandilya, D.K., Israni, R., Joseph, P.E., Kumar, A. and Pillai, M. (2017) Identification of Oxidative Degradation Products of Lansoprazole by Using High Resolution Mass Spectrometry Spectral Data. International Journal of Analytical Mass Spectrometry and Chromatography, 5, 57-69.

http://dx.doi.org/10.4236/ijamsc.2017.53004

Received: April 25, 2017

Accepted: September 10, 2017

Published: September 13, 2017

Copyright $\odot 2017$ by authors and Scientific Research Publishing Inc. This work is licensed under the Creative Commons Attribution International License (CC BY 4.0).

http://creativecommons.org/licenses/by/4.0/

\begin{abstract}
The study focused on the application of high-resolution mass spectrometry for the identification of impurities in pharmaceutical small molecules. A high-performance liquid chromatography (HPLC) coupled high resolution mass spectrometer (HRMS) was used for identification of oxidative degradation impurities (DIs) of lansoprazole. The utilization of HRMS facilitates to determine the accurate mass of impurities and their fragment/product ions. A fast mass spectrometer (MS) compatible reverse phase chromatography method was used to investigate the oxidative stressed impurities. HPLC column; C18 $(50 \times 4.6 \mathrm{~mm}, 3.5 \mu \mathrm{m})$ was used with gradient elution. Spectral data acquired using information dependent acquisition (IDA) with real time dynamic background subtraction algorithm (DBS). Three oxidative impurities: DI-I ( $\mathrm{m} / \mathrm{z}$ 386.0781), DI-II ( $\mathrm{m} / \mathrm{z} 402.0734)$ and DI-III $(\mathrm{m} / \mathrm{z} 386.0785)$, was observed during this study; interpretation of high resolution spectral data of all three impurities was carried out; elemental composition and molecular structure was proposed for major fragments. In this study mass error was found $\leq 7.7$ parts per million ( $\mathrm{ppm})$.
\end{abstract}

\section{Keywords}

Lansoprazole, Oxidative Degradation Products of Lansoprazole

\section{Introduction}

Structural analysis of degradation impurities is one of the essential studies in pharmaceutical analysis, particularly during the product development process [1]. The safety of any drug product is not only depending on the toxicological 
properties of the active drug substance, but also on the impurities present in it; monitoring and the control of degradation impurities in pharmaceuticals is a key element of the guidelines issued by the International Council for Harmonization of Technical Requirements for Pharmaceuticals for Human Use (ICH) [2] [3] [4].

There are many analytical techniques which are commonly being used for impurity profiling, high pressure liquid chromatography (HPLC) coupled with ultraviolet (UV) or photo diode array (PDA) detector is one of the most common analytical techniques to estimate the degradation impurities. But the identification and structural confirmation of the degradation products is done by using MS detectors i.e. Triple quadrupole, ion trap, and high resolution mass spectrometers (time of flight and orbitrap) [5] [6] [7]. For this study time of flight high-resolution mass analyzer selected; use of high resolution mass spectrometer facilitates accurate measurement of $\mathrm{m} / \mathrm{z}$ values, which supports to propose the exact elemental compositions and to predict the structure of parent and product ions [8] [9] [10] [11]. Oxidation sensitive drug molecule lansoprazole was selected for this study. Lansoprazole [12] [13] [14] belongs to a group of drugs called proton pump inhibitors, which inhibits the stomach's production of gastric acids. Physically, it is a white to brownish-white odorless crystalline powder and chemically known as

2-[[[3-Methyl-4-(2,2,2-trifluoroethoxy)-2pyridyl]-methyl-]sulfinyl]benzimidazol e. Its empirical formula is $\mathrm{C}_{16} \mathrm{H}_{14} \mathrm{~F}_{3} \mathrm{~N}_{3} \mathrm{O}_{2} \mathrm{~S}$ with a molecular mass of 369.363 $\mathrm{g} / \mathrm{mol}$ and its monoisotopic molecular weight is 369.0759 . Molecular structure and exact mass of lansoprazole molecule is presented in Figure 1.

The goal of present study was identification of the oxidative degradation impurities of lansoprazole using high-resolution MS and MS/MS analysis, two oxidation impurities (USP impurities-"N-Oxide" and "related compound A") of lansoprazole are specified impurity in US pharmacopeia [15] and other impurities discussed in several other research publications [16] [17] [18]; during this study one unspecified oxidative impurity (di-oxidized) with $\mathrm{m} / \mathrm{z} 402.0734$ was observed; high-resolution mass spectral data of lansoprazole and its oxidative

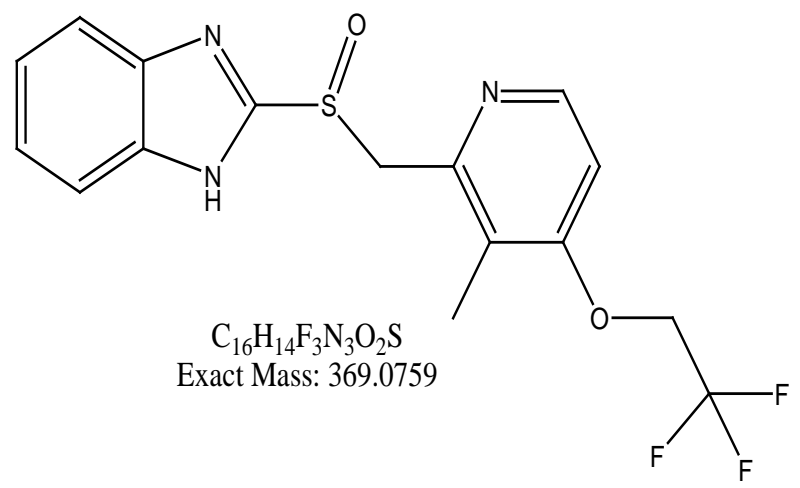

Figure 1. Molecular structure and exact mass of lansoprazole molecules. 
impurities generated using electrospray ionization and collision induced dissociation. Followed by interpretation of spectral data using basic interpretation rules and workflow [19] [20]; elemental composition, molecular structures proposed and mass error calculated for major $\mathrm{m} / \mathrm{z}$ values.

\section{Experiment}

\subsection{Material}

The ultrapure water (18.2 M $\Omega$ ) was obtained using MilliQ apparatus; manufactured by Millipore, USA. Mass spectrometry grade solvents methanol and acetonitrile; manufactured by J.T. Baker, USA. meta-chloroperbenzoic acid (mCPBA); manufactured by Sigma-Aldrich, USA. Ammonium acetate and sodium hydroxide; manufactured by Merck, India. The lansoprazole was extracted from commercially available generic dosage form; manufactured by Intas Pharmaceutical, India. Powdered and finally extracted in diluent acetonitrile, methanol and $0.1 \mathrm{~N}$ sodium hydroxide, (5:2:3). The solution was centrifuged and supernatant was subjected to degradation study.

\subsection{Instruments and Conditions}

Prominence 20AD HPLC (from Shimadzu corporation, Kyoto, Japan) with UV detector was connected with the AB SCIEX Triple TOF $^{\mathrm{TM}} 5600$ (form AB SCIEX, Concord, $\mathrm{ON}$ ) high resolution mass spectrometer used, for the identification of the drug and its degradation impurities.

Elution of the degradation impurities was achieved on Gemini-NX C18 $(50 \times$ $4.6 \mathrm{~mm}, 3.5 \mu \mathrm{m}$ ) column (from Phenomenex, USA) at the flow rate of 1.0 $\mathrm{mL} / \mathrm{min}$. The sample tray temperature was $15^{\circ} \mathrm{C}$ and column oven temperature was $45^{\circ} \mathrm{C}$. The chromatographic gradient elution conditions were used; $5 \mathrm{mM}$ ammonium acetate and methanol in ratio of 95:5 (mobile phase A) and acetonitrile and methanol in a ratio of 1:1 (mobile phase $\mathrm{B}$ ). The mobile phase gradient was started at $0 \mathrm{~min} 20 \%$ (B pump concentration) and end at $9 \mathrm{~min} / 70 \%$ (B pump concentration) gradient program reproduced from [16]; UV detector was set to $285 \mathrm{~nm}$. Injection volume was kept $5 \mu \mathrm{L}$. Total run time was set to $11 \mathrm{~min}$. The flow rate was splited after the HPLC column in the ratio of $1 / 10$, producing an inlet flow into the mass spectrometer was about $0.10 \mathrm{~mL} / \mathrm{min}$.

Oxidative stress of lansoprazole was carried out using about $1 \mathrm{mg} / \mathrm{mL} w / \mathrm{v}$ solution of oxidant m-CPBA in acetonitrile for 20 minutes. After stressed exposure sample was reconstituted with diluent and final concentration of lansoprazole in sample solution was about $400 \mu \mathrm{g} / \mathrm{mL}$. Injected to the mass spectrometry using liquid chromatography system. All the data acquisition was performed with a Triple TOF ${ }^{\mathrm{TM}} 5600$ System (AB SCIEX, Concord, ON) coupled with dual ionization source (AB SCIEX, Concord, $\mathrm{ON}$ ) which has electrospray and atmospheric pressure chemical ionization (APCI) probe (from AB SCIEX, Concord, ON) for analysis. All the experiments were carried out in electrospray positive ionization mode in mass spectrometer. Data was acquired using optimized mass spectro- 
meter parameters; an ion spray voltage of $+5.5 \mathrm{kV}$, curtain gas of $25 \mathrm{PSI}$, nebulizer gas of 50 PSI, and an interface heater temperature of $600^{\circ} \mathrm{C}$ with drying gas (GS2) 50 PSI. Collision energy (CE) setting of $30 \mathrm{~V}$ with a spread of $\pm 10 \mathrm{~V}$ was applied to all parent ions for collisional induced dissociation (CID). Real time dynamic background subtraction algorithm was switched on during the IDA acquisition to eliminate the background noise [21]. Acquired data were processed using Analyst TF ${ }^{\circledast}$ 1.5, PeakView ${ }^{\circledast}$ 1.1.1. (from AB SCIEX, Concord, ON).

\section{Result and Discussion}

Three oxidative degradation products/impurities of lansoprazole (DS) was identified using HRMS. Simultaneous acquisition of MS and MS/MS data was developed using non-targeted generic (IDA) method with real time background subtraction. All three degradation impurities of oxidative stressed condition were identified using full scan mass spectra and their product ion data at chromatography scale, lansoprazole (DS) peak was observed at $5.5 \mathrm{~min} ; \mathrm{m} / \mathrm{z} 370.0836$ and degradation impurities were observed at retention time $4.3 \mathrm{~min} ; \mathrm{m} / z$ 386.0781 (DI-I), at $4.4 \mathrm{~min}$ in the tailing of DI-I peak; $\mathrm{m} / z 402.0734$ (DI-II) and third degradation product DI-III; $\mathrm{m} / z 386.0785$ was observed at $5.40 \mathrm{~min}$ in the fronting of lansoprazole (DS) peak. LC-UV chromatograms of (as such extracted DS), m-CPBA blank and oxidative stressed sample presented in Figures $2(a)-(e)$

The full scan MS spectra of lansoprazole (DS), DI-I, DI-II and DI-III as presented in Figure 3, extracted ion chromatogram (XIC) of DI-I, DI-II and DI-III presented in Figure 4. Product ion spectra (HR-MS/MS) of lansoprazole, DI-I, DI-II and DI-III were obtained from information dependent information (IDA) experiments as presented in Figures 5-8 respectively. Interpretation was carried out by utilizing workflow [19] and basic interpretation rules.

Structure confirmation and comparison with the spectral data of lansoprazole (DS) was carried out for all three impurities, accurate $\mathrm{m} / \mathrm{z}$ values helped to predict the molecular structures of impurities, elemental compositions and molecular structure of product ions. Interpretation data summarized in Figures 5-9 and Tables 1-5.

The mass spectral data interpretation summarized as follows; experimental $\mathrm{m} / \mathrm{z}$ value for lansoprazole molecular ion peak was $370.0836[\mathrm{M}+\mathrm{H}]^{+}$. The TOF MS/MS spectrum of lansoprazole also exhibited molecular ion $\mathrm{m} / \mathrm{z} 370.0842$ as $[\mathrm{M}+\mathrm{H}]^{+}$(calculated formula $\mathrm{C}_{16} \mathrm{H}_{15} \mathrm{~F}_{3} \mathrm{~N}_{3} \mathrm{O}_{2} \mathrm{~S}^{+}$, exact mass 370.0832 , mass error $2.7 \mathrm{ppm}$ ). Molecular ion further fragmented into nine major fragments; $\mathrm{m} / \mathrm{z}$ 352.0731, 252.0304, 235.0274, 234.0198, 205.0713, 204.0634, 190.0472, 136.0761, and 119.0609 .

The interpretation of fragments or product ions summarized in Table 2 and Figure 5; fragment ion 352.0731 (calculated formula $\mathrm{C}_{16} \mathrm{H}_{13} \mathrm{~F}_{3} \mathrm{~N}_{3} \mathrm{OS}^{+}$, exact mass 352.0726, mass error $1.4 \mathrm{ppm}$ ), fragment ion 252.0304 (calculated formula $\mathrm{C}_{9} \mathrm{H}_{9} \mathrm{~F}_{3} \mathrm{NO}_{2} \mathrm{~S}^{+}$, exact mass 252.07301, mass error $1.2 \mathrm{ppm}$ ), fragment ion 


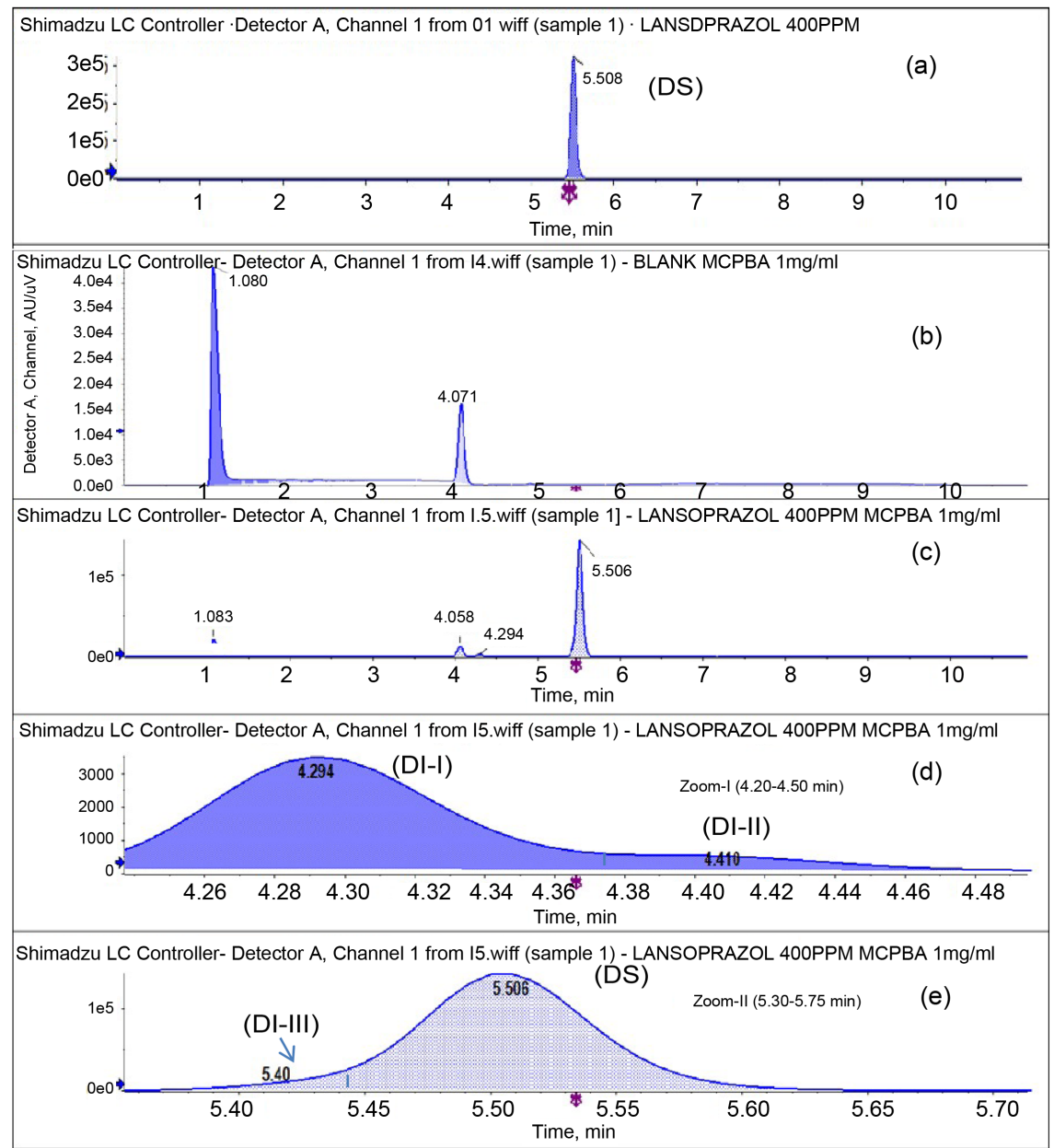

Figure 2. (a) Unstressed UV chromatogram of lansoprazole, b: m-CPBA blank (1 and 4 min retention time peaks from $\mathrm{m}$-CPBA); (c) Oxidative stress LC-UV chromagram (d) zoom LC-UV chromatogram showing DI-II:4.3 min and DI-II 4.4 retention time); (e) zoom LC-UV chromatogram showing DI-III : $5.4 \mathrm{~min}$ and DS 5.5 retention time.

Table 1. MS spectral analysis of lansoprazole (DS), DI-I, DI-II and DI-III.

\begin{tabular}{cccccccc}
\hline ID & $\begin{array}{c}\text { MS } \\
\mathrm{t}_{\mathrm{R}}(\mathrm{min})\end{array}$ & $\begin{array}{c}\mathbf{m} / \boldsymbol{z} \text { value } \\
(\mathrm{MS})\end{array}$ & Ion type & $\begin{array}{c}\Delta \text { Da } \\
\text { with DS } \\
\mathbf{m} / \boldsymbol{z}\end{array}$ & $\begin{array}{c}\text { Calculated } \\
\text { Elemental } \\
\text { composition }\end{array}$ & $\begin{array}{c}\text { Calculated Error } \\
\text { Mass }(\boldsymbol{m} / \mathbf{z})\end{array}$ & $\mathrm{ppm}$ \\
\hline $\begin{array}{c}\text { Lansoprazole } \\
\text { (DS) }\end{array}$ & 5.5 & 370.0836 & {$[\mathrm{M}+\mathrm{H}]^{+}$} & $\mathrm{N} / \mathrm{A}$ & $\mathrm{C}_{16} \mathrm{H}_{15} \mathrm{~F}_{3} \mathrm{~N}_{3} \mathrm{O}_{2} \mathrm{~S}^{+}$ & 370.0832 & 1.1 \\
DI-I & 4.3 & 386.0781 & {$[\mathrm{M}+\mathrm{H}]^{+}$} & 15.9945 & $\mathrm{C}_{16} \mathrm{H}_{15} \mathrm{~F}_{3} \mathrm{~N}_{3} \mathrm{O}_{3} \mathrm{~S}^{+}$ & 386.0781 & 0.0 \\
DI-II & 4.4 & 402.0734 & {$[\mathrm{M}+\mathrm{H}]^{+}$} & 31.9898 & $\mathrm{C}_{16} \mathrm{H}_{15} \mathrm{~F}_{3} \mathrm{~N}_{3} \mathrm{O}_{4} \mathrm{~S}^{+}$ & 402.0730 & 1.0 \\
DI-III & 5.4 & 386.0785 & {$[\mathrm{M}+\mathrm{H}]^{+}$} & 15.9949 & $\mathrm{C}_{16} \mathrm{H}_{15} \mathrm{~F}_{3} \mathrm{~N}_{3} \mathrm{O}_{3} \mathrm{~S}^{+}$ & 386.0781 & 1.0 \\
\hline
\end{tabular}

${ }^{*}$ Mass error $=$ difference between measured accurate mass and calculated accurate mass/calculated accurate mass $\times 10^{6}$. N/A: not applicable.

235.0274 (calculated formula $\mathrm{C}_{9} \mathrm{H}_{8} \mathrm{~F}_{3} \mathrm{NOS}^{*+}$, exact mass 235.0273, mass error 0.4 ppm), fragment ion 234.0198 (calculated formula $\mathrm{C}_{9} \mathrm{H}_{7} \mathrm{~F}_{3} \mathrm{NOS}^{\circ+}$, exact mass 234.0195, mass error $1.3 \mathrm{ppm}$ ) fragment ion 205.0713 (calculated formula $\mathrm{C}_{9} \mathrm{H}_{10} \mathrm{~F}_{3} \mathrm{NO}^{\circ}$, exact mass 205.0709 , mass error $2.0 \mathrm{ppm}$ ), fragment ion 204.0634 


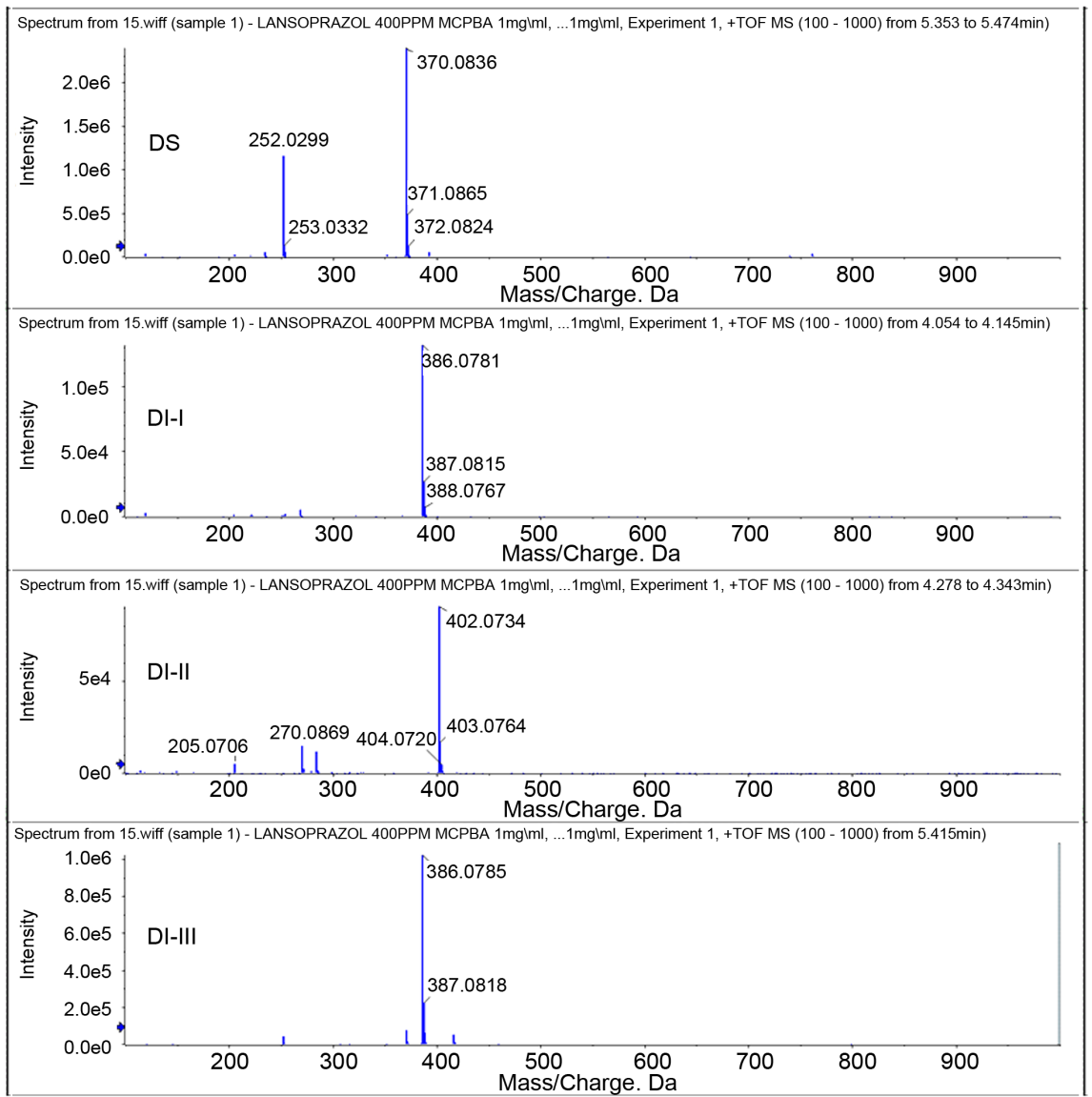

Figure 3. Parent ion (HR-MS) spectra of lansoprazole (DS), degradation impurity-I (DII), degradation impurity-II (DI-II) and degradation impurity-III (DI-III).

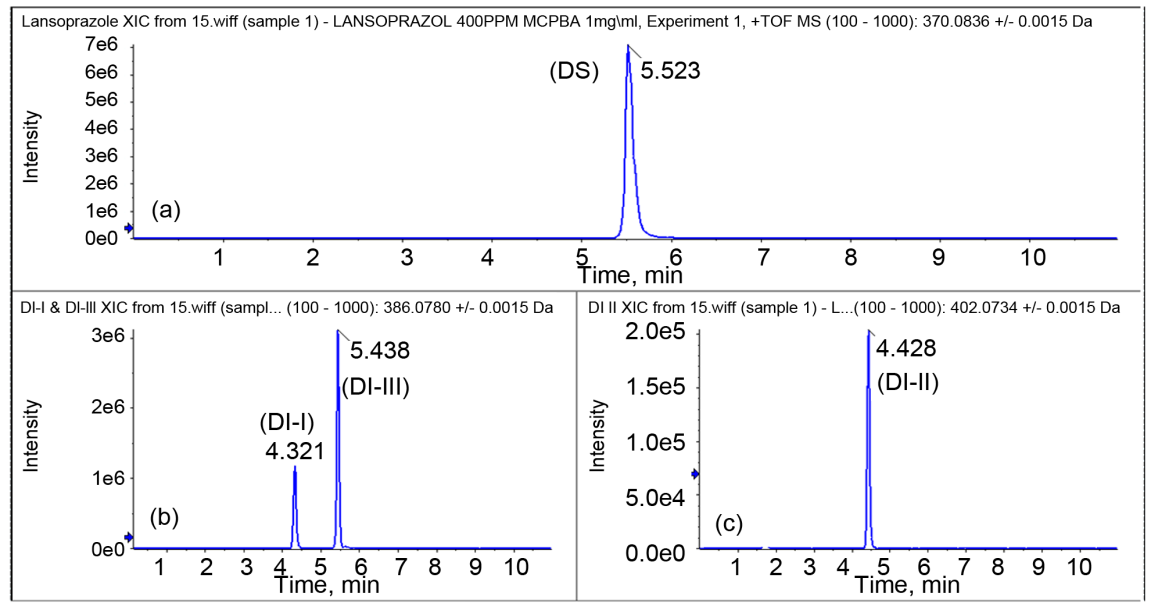

Figure 4. (a) Extracted ion chromatogram of $\mathrm{m} / \mathrm{z}$ 370.0836(lansoprazole-DS); (b) $\mathrm{m} / \mathrm{z}$ 386.0780 (DI-I $4.3 \mathrm{~min}$, DI-III $5.4 \mathrm{~min}$ retention time); (c) 402.07 (DI-II: $4.4 \mathrm{~min}$ retention time).

(calculated formula $\mathrm{C}_{9} \mathrm{H}_{9} \mathrm{~F}_{3} \mathrm{NO}^{+}$, exact mass 204.0631, mass error $1.5 \mathrm{ppm}$ ), fragment ion 190.0472, (calculated formula $\mathrm{C}_{8} \mathrm{H}_{7} \mathrm{~F}_{3} \mathrm{NO}^{+}$, exact mass 190.0474, mass error $-1.1 \mathrm{ppm}$ ), fragment ion 136.0761 (calculated formula $\mathrm{C}_{8} \mathrm{H}_{10} \mathrm{NO}^{+}$, ex- 
Table 2. MS/MS spectral analysis of lansoprazole (DS).

\begin{tabular}{|c|c|c|c|c|c|c|}
\hline $\begin{array}{c}\text { Measured } \\
\text { Mass }(m / z)\end{array}$ & $\begin{array}{c}\text { Nitrogen } \\
\text { Rule }\end{array}$ & $\begin{array}{c}\text { No. of } \\
\text { Nitrogen }(s)^{*}\end{array}$ & $\begin{array}{l}\text { Proposed } \\
\text { Formula }\end{array}$ & $\begin{array}{c}\text { Electron } \\
\text { Paring }\end{array}$ & $\begin{array}{c}\text { Calculated } \\
\text { Mass }(m / z)\end{array}$ & $\begin{array}{c}\text { Error** } \\
(\mathrm{ppm})\end{array}$ \\
\hline 370.0842 & $\mathrm{ON}$ & 3 & $\mathrm{C}_{16} \mathrm{H}_{15} \mathrm{~F}_{3} \mathrm{~N}_{3} \mathrm{O}_{2} \mathrm{~S}^{+}$ & {$[\mathrm{M}+\mathrm{H}]^{+}$} & 370.0832 & 2.7 \\
\hline 352.0731 & $\mathrm{ON}$ & 3 & $\mathrm{C}_{16} \mathrm{H}_{13} \mathrm{~F}_{3} \mathrm{~N}_{3} \mathrm{OS}^{+}$ & $\mathrm{EE}$ & 352.0726 & 1.4 \\
\hline 252.0304 & $\mathrm{ON}$ & 1 & $\mathrm{C}_{9} \mathrm{H}_{9} \mathrm{~F}_{3} \mathrm{NO}_{2} \mathrm{~S}^{+}$ & $\mathrm{EE}$ & 252.0301 & 1.2 \\
\hline 235.0274 & ON & 1 & $\mathrm{C}_{9} \mathrm{H}_{8} \mathrm{~F}_{3} \mathrm{NOS}^{\bullet+}$ & $\mathrm{OE}$ & 235.0273 & 0.4 \\
\hline 234.0198 & $\mathrm{ON}$ & 1 & $\mathrm{C}_{9} \mathrm{H}_{7} \mathrm{~F}_{3} \mathrm{NOS}^{\bullet+}$ & $\mathrm{OE}$ & 234.0195 & 1.3 \\
\hline 205.0713 & $\mathrm{ON}$ & 1 & $\mathrm{C}_{9} \mathrm{H}_{10} \mathrm{~F}_{3} \mathrm{NO}^{\bullet+}$ & $\mathrm{OE}$ & 205.0709 & 2.0 \\
\hline 204.0634 & $\mathrm{ON}$ & 1 & $\mathrm{C}_{9} \mathrm{H}_{9} \mathrm{~F}_{3} \mathrm{NO}^{+}$ & $\mathrm{EE}$ & 204.0631 & 1.5 \\
\hline 190.0472 & $\mathrm{ON}$ & 1 & $\mathrm{C}_{8} \mathrm{H}_{7} \mathrm{~F}_{3} \mathrm{NO}^{+}$ & $\mathrm{EE}$ & 190.0474 & -1.1 \\
\hline 136.0761 & $\mathrm{ON}$ & 1 & $\mathrm{C}_{8} \mathrm{H}_{10} \mathrm{NO}^{+}$ & $\mathrm{EE}$ & 136.0757 & 2.9 \\
\hline 119.0609 & EN & 2 & $\mathrm{C}_{7} \mathrm{H}_{7} \mathrm{~N}_{2}^{+}$ & $\mathrm{EE}$ & 119.0604 & 4.2 \\
\hline
\end{tabular}

EE: even electron; OE: Odd electron; ON: odd nitrogen; EN: even nitrogen. ${ }^{*}$ Number of nitrogen prediction, is based on the structure of parent/lansoprazole (DS), nitrogen rule and electron paring. ${ }^{* *}$ Mass error $=$ difference between measured accurate mass and calculated accurate mass/calculated accurate mass $\times 10^{6}$.

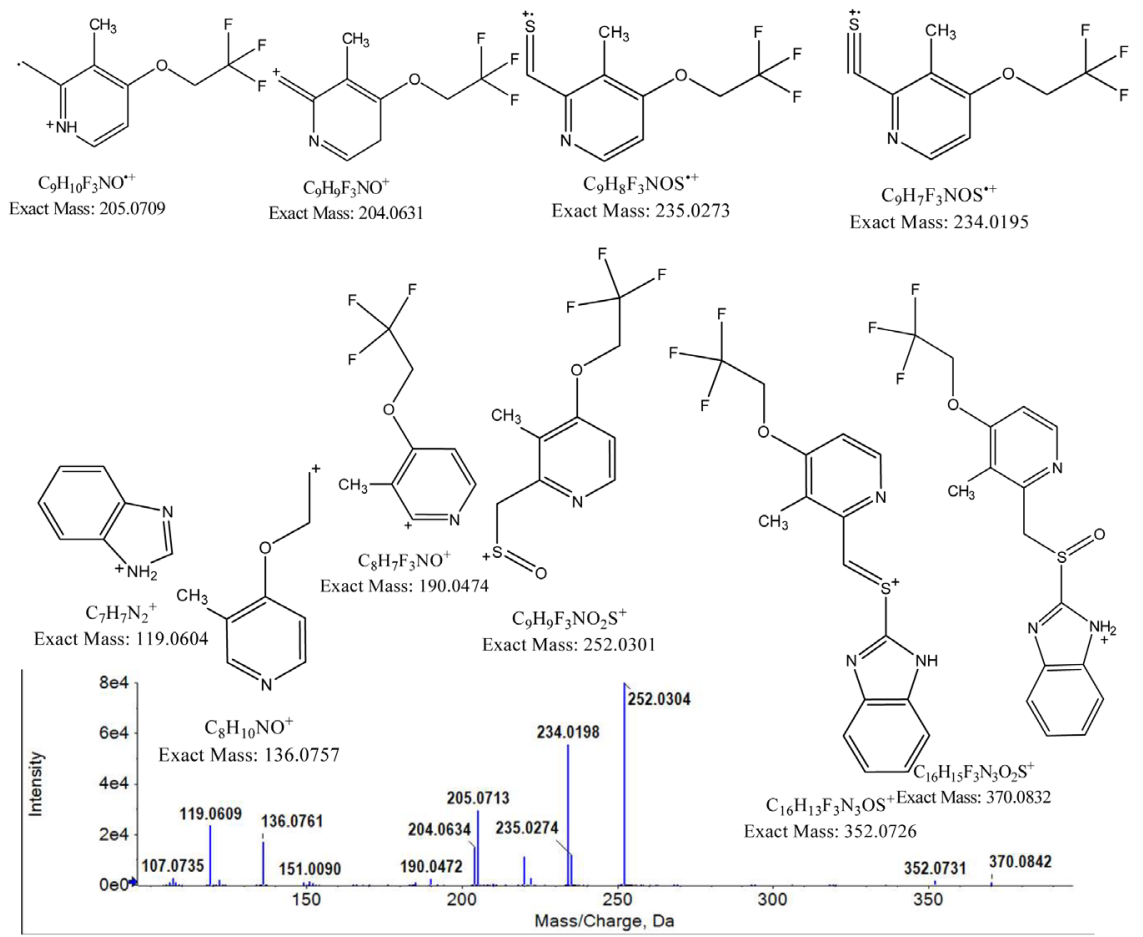

Figure 5. MS/MS spectrum of lansoprazole, molecular structue of fragment ions along with elemental composition and exact mass.

act mass 136.0757, mass error $2.9 \mathrm{ppm}$ ) and 119.0609 (calculated formula $\mathrm{C}_{7} \mathrm{H}_{7} \mathrm{~N}_{2}{ }^{+}$, exact mass 119.0604 , mass error $4.2 \mathrm{ppm}$ )

In the Full scan spectrum of DI-I molecular ion peak was $386.0781[\mathrm{M}+\mathrm{H}]^{+}$. The TOF MS/MS spectrum of DI-I also exhibited molecular ion $\mathrm{m} / z 386.0781$ $\mathrm{Da}$ as $[\mathrm{M}+\mathrm{H}]^{+}$(calculated formula $\mathrm{C}_{16} \mathrm{H}_{15} \mathrm{~F}_{3} \mathrm{~N}_{3} \mathrm{O}_{3} \mathrm{~S}^{+}$, exact mass 386.0781 , mass error $0 \mathrm{ppm}$ ), molecular ion further fragmented into seven major fragments, the 
$m / z$ 268.0256, 250.0148, 220.0581, 204.0636, 190.0472, 152.0704 and 119.0605 . The proposed interpretation of fragments summarized in Table 3 and Figure 6; fragment ion 268.0256 (calculated formula $\mathrm{C}_{9} \mathrm{H}_{9} \mathrm{~F}_{3} \mathrm{NO}_{3} \mathrm{~S}^{+}$, exact mass

Table 3. MS/MS spectral analysis of DI-I.

\begin{tabular}{|c|c|c|c|c|c|c|}
\hline $\begin{array}{l}\text { Measured } \\
\text { Mass }(m / z)\end{array}$ & $\begin{array}{c}\text { Nitrogen } \\
\text { Rule }\end{array}$ & $\begin{array}{c}\text { No. of } \\
\text { Nitrogen }(s)^{*}\end{array}$ & $\begin{array}{l}\text { Proposed } \\
\text { Formula }\end{array}$ & $\begin{array}{c}\text { Electron } \\
\text { Paring }\end{array}$ & $\begin{array}{c}\text { Calculated Mass } \\
\qquad(m / z)\end{array}$ & $\begin{array}{l}\text { Error }^{* *} \\
\text { (ppm) }\end{array}$ \\
\hline 386.0781 & ON & 3 & $\mathrm{C}_{16} \mathrm{H}_{15} \mathrm{~F}_{3} \mathrm{~N}_{3} \mathrm{O}_{3} \mathrm{~S}^{+}$ & {$[\mathrm{M}+\mathrm{H}]^{+}$} & 386.0781 & 0.0 \\
\hline 268.0256 & ON & 1 & $\mathrm{C}_{9} \mathrm{H}_{9} \mathrm{~F}_{3} \mathrm{NO}_{3} \mathrm{~S}^{+}$ & $\mathrm{EE}$ & 268.0250 & 2.2 \\
\hline 250.0148 & ON & 1 & $\mathrm{C}_{9} \mathrm{H}_{7} \mathrm{~F}_{3} \mathrm{NO}_{2} \mathrm{~S}^{+}$ & $\mathrm{EE}$ & 250.0144 & 1.6 \\
\hline 220.0581 & ON & 1 & $\mathrm{C}_{9} \mathrm{H}_{9} \mathrm{~F}_{3} \mathrm{NO}_{2}^{+}$ & $\mathrm{EE}$ & 220.0580 & 0.5 \\
\hline 204.0636 & ON & 1 & $\mathrm{C}_{9} \mathrm{H}_{9} \mathrm{~F}_{3} \mathrm{NO}^{+}$ & $\mathrm{EE}$ & 204.0631 & 2.5 \\
\hline 190.0472 & ON & 1 & $\mathrm{C}_{8} \mathrm{H}_{7} \mathrm{~F}_{3} \mathrm{NO}^{+}$ & $\mathrm{EE}$ & 190.0474 & -1.1 \\
\hline 152.0704 & ON & 1 & $\mathrm{C}_{8} \mathrm{H}_{10} \mathrm{NO}_{2}+$ & $\mathrm{EE}$ & 152.0706 & -1.3 \\
\hline 119.0605 & EN & 2 & $\mathrm{C}_{7} \mathrm{H}_{7} \mathrm{~N}_{2}^{+}$ & $\mathrm{EE}$ & 119.0604 & 0.8 \\
\hline
\end{tabular}

EE: even electron; ON: odd nitrogen; EN: even nitrogen. ${ }^{*}$ Number of nitrogen prediction, is based on the structure of parent/lansoprazole (DS), nitrogen rule and electron paring. ${ }^{* \star}$ Mass error $=$ difference between measured accurate mass and calculated accurate mass/calculated accurate mass $\times 10^{6}$.

Table 4. MS/MS spectral analysis of DI-II.

\begin{tabular}{ccccccc}
\hline $\begin{array}{c}\text { Measured } \\
\text { Mass }(\mathrm{m} / \mathrm{z})\end{array}$ & $\begin{array}{c}\text { Nitrogen } \\
\text { Rule }\end{array}$ & $\begin{array}{c}\text { No. of } \\
\text { Nitrogen }(\mathrm{s})^{*}\end{array}$ & $\begin{array}{c}\text { Proposed } \\
\text { Formula }\end{array}$ & $\begin{array}{c}\text { Electron } \\
\text { Paring }\end{array}$ & $\begin{array}{c}\text { Calculated } \\
\text { Mass }(\mathrm{m} / \mathbf{z})\end{array}$ & $\begin{array}{c}\text { Error }^{* *} \\
(\mathrm{ppm})\end{array}$ \\
\hline 402.0739 & $\mathrm{ON}$ & 3 & $\mathrm{C}_{16} \mathrm{H}_{15} \mathrm{~F}_{3} \mathrm{~N}_{3} \mathrm{O}_{4} \mathrm{~S}^{+}$ & {$[\mathrm{M}+\mathrm{H}]^{+}$} & 402.0730 & 2.2 \\
284.0204 & $\mathrm{ON}$ & 1 & $\mathrm{C}_{9} \mathrm{H}_{9} \mathrm{~F}_{3} \mathrm{NO}_{4} \mathrm{~S}^{+}$ & $\mathrm{EE}$ & 284.0199 & 1.8 \\
220.0582 & $\mathrm{ON}$ & 1 & $\mathrm{C}_{9} \mathrm{H}_{9} \mathrm{~F}_{3} \mathrm{NO}_{2}^{+}$ & $\mathrm{EE}$ & 220.0580 & 0.9 \\
190.0477 & $\mathrm{ON}$ & 1 & $\mathrm{C}_{8} \mathrm{H}_{7} \mathrm{~F}_{3} \mathrm{NO}^{+}$ & $\mathrm{EE}$ & 190.0474 & 1.6 \\
119.0610 & $\mathrm{EN}$ & 2 & $\mathrm{C}_{7} \mathrm{H}_{7} \mathrm{~N}_{2}^{+}$ & $\mathrm{EE}$ & 119.0604 & 5.0 \\
\hline
\end{tabular}

EE: even electron; ON: odd nitrogen; EN: even nitrogen. ${ }^{\star}$ Number of nitrogen prediction, is based on the structure of parent, lansoprazole (DS), nitrogen rule and electron paring. ${ }^{* \star}$ Mass error $=$ difference between measured accurate mass and calculated accurate mass/calculated accurate mass $\times 10^{6}$.

Table 5. MS/MS spectral analysis of DI-III.

\begin{tabular}{|c|c|c|c|c|c|c|}
\hline $\begin{array}{c}\text { Measured } \\
\text { Mass }(m / z)\end{array}$ & $\begin{array}{c}\text { Nitrogen } \\
\text { Rule }\end{array}$ & $\begin{array}{c}\text { No. of } \\
\text { Nitrogen }(s)^{*}\end{array}$ & $\begin{array}{l}\text { Proposed } \\
\text { Formula }\end{array}$ & $\begin{array}{c}\text { Electron } \\
\text { Paring }\end{array}$ & $\begin{array}{l}\text { Calculated Mass } \\
\qquad(\mathrm{m} / \mathrm{z})\end{array}$ & $\begin{array}{l}\text { Error }^{* *} \\
(\mathrm{ppm})\end{array}$ \\
\hline 386.0783 & $\mathrm{ON}$ & 3 & $\mathrm{C}_{16} \mathrm{H}_{15} \mathrm{~F}_{3} \mathrm{~N}_{3} \mathrm{O}_{3} \mathrm{~S}^{+}$ & {$[\mathrm{M}+\mathrm{H}]^{+}$} & 386.0781 & 0.5 \\
\hline 322.1168 & $\mathrm{ON}$ & 3 & $\mathrm{C}_{16} \mathrm{H}_{15} \mathrm{~F}_{3} \mathrm{~N}_{3} \mathrm{O}^{+}$ & $\mathrm{EE}$ & 322.1162 & 1.9 \\
\hline 268.0260 & ON & 1 & $\mathrm{C}_{9} \mathrm{H}_{9} \mathrm{~F}_{3} \mathrm{NO}_{3} \mathrm{~S}^{+}$ & $\mathrm{EE}$ & 268.0250 & 3.7 \\
\hline 222.0753 & ON & 1 & $\mathrm{C}_{9} \mathrm{H}_{11} \mathrm{~F}_{3} \mathrm{NO}_{2}+$ & $\mathrm{EE}$ & 222.0736 & 7.7 \\
\hline 220.0585 & $\mathrm{ON}$ & 1 & $\mathrm{C}_{9} \mathrm{H}_{9} \mathrm{~F}_{3} \mathrm{NO}_{2}+$ & $\mathrm{EE}$ & 220.0580 & 2.3 \\
\hline 205.0715 & $\mathrm{ON}$ & 1 & $\mathrm{C}_{9} \mathrm{H}_{10} \mathrm{~F}_{3} \mathrm{NO}^{\cdot+}$ & $\mathrm{OE}$ & 205.0709 & 2.9 \\
\hline 204.0635 & ON & 1 & $\mathrm{C}_{9} \mathrm{H}_{9} \mathrm{~F}_{3} \mathrm{NO}^{+}$ & $\mathrm{EE}$ & 204.0631 & 2.0 \\
\hline 190.0475 & ON & 1 & $\mathrm{C}_{8} \mathrm{H}_{7} \mathrm{~F}_{3} \mathrm{NO}^{+}$ & $\mathrm{EE}$ & 190.0474 & 0.5 \\
\hline 136.0759 & $\mathrm{ON}$ & 1 & $\mathrm{C}_{8} \mathrm{H}_{10} \mathrm{NO}^{+}$ & $\mathrm{EE}$ & 136.0757 & 1.5 \\
\hline 119.0605 & EN & 2 & $\mathrm{C}_{7} \mathrm{H}_{7} \mathrm{~N}_{2}^{+}$ & $\mathrm{EE}$ & 119.0604 & 0.8 \\
\hline
\end{tabular}

EE: even electron; OE: Odd electron; ON: odd nitrogen; EN: even nitrogen. ${ }^{*}$ Number of nitrogen prediction, is based on the structure of parent, lansoprazole (DS), nitrogen rule and electron paring. ${ }^{*}$ Mass error $=$ difference between measured accurate mass and calculated accurate mass/calculated accurate mass $\backslash 10^{6}$. 
268.0250, mass error $2.2 \mathrm{ppm}$ ), fragment ion 250.0148 (calculated formula $\mathrm{C}_{9} \mathrm{H}_{7} \mathrm{~F}_{3} \mathrm{NO}_{2} \mathrm{~S}^{+}$, exact mass 250.0144 , mass error $1.6 \mathrm{ppm}$ ), fragment ion 220.0581 (calculated formula $\mathrm{C}_{9} \mathrm{H}_{9} \mathrm{~F}_{3} \mathrm{NO}_{2} \mathrm{~S}^{+}$, exact mass 220.580 , mass error $0.5 \mathrm{ppm}$ ), fragment ion 204.0636 (calculated formula $\mathrm{C}_{9} \mathrm{H}_{9} \mathrm{~F}_{3} \mathrm{NO}^{+}$, exact mass 204.0631, mass error $2.5 \mathrm{ppm}$ ), fragment ion 190.0472, (calculated formula $\mathrm{C}_{8} \mathrm{H}_{7} \mathrm{~F}_{3} \mathrm{NO}^{+}$, exact mass 190.0474, mass error $-1.1 \mathrm{ppm}$ ), fragment ion 152.0704 (calculated formula $\mathrm{C}_{8} \mathrm{H}_{10} \mathrm{NO}_{2}^{+}$, exact mass 152.0706 , mass error $-1.3 \mathrm{ppm}$ ) and 119.0605 (calculated formula $\mathrm{C}_{7} \mathrm{H}_{7} \mathrm{~N}_{2}{ }^{+}$, exact mass 119.0604 , mass error $0.8 \mathrm{ppm}$ ). The presence of $m / z 152.0704$ in product ion spectra (refer Table 3 and Figure 6) further confirms DI-I as USP N-oxide impurity; refer Figure 9.

In full scan spectrum of DI-II, molecular ion peak was found as 402.0734 $[\mathrm{M}+\mathrm{H}]^{+}$(refer Figure 3 ). The TOF MS/MS spectrum of DI-II also exhibit the molecular ion $\mathrm{m} / z 402.0739$ as $[\mathrm{M}+\mathrm{H}]^{+}$(calculated formula $\mathrm{C}_{16} \mathrm{H}_{15} \mathrm{~F}_{3} \mathrm{~N}_{3} \mathrm{O}_{4} \mathrm{~S}^{+}$, exact mass 402.0730, mass error $2.2 \mathrm{ppm}$ ), molecular ion further fragmented into four major fragments, the $m / z 284.0204,220.0582,190.0477$ and 119.0610 .

The interpretation of fragments summarized in Table 4 and Figure 7; fragment ion 284.0204 (calculated formula $\mathrm{C}_{9} \mathrm{H}_{9} \mathrm{~F}_{3} \mathrm{NO}_{4} \mathrm{~S}^{+}$, exact mass 284.0199 , mass error $1.8 \mathrm{ppm}$ ), fragment ion 220.0582 (calculated formula $\mathrm{C}_{9} \mathrm{H}_{9} \mathrm{~F}_{3} \mathrm{NO}_{2}^{+}$, exact mass 220.0580, mass error $0.9 \mathrm{ppm}$ ), fragment ion 190.0477, (calculated formula $\mathrm{C}_{8} \mathrm{H}_{7} \mathrm{~F}_{3} \mathrm{NO}^{+}$, exact mass 190.0474 , mass error $1.6 \mathrm{ppm}$ ), and 119.0610 (calculated formula $\mathrm{C}_{7} \mathrm{H}_{7} \mathrm{~N}_{2}^{+}$exact mass 119.0604 , mass error $5.0 \mathrm{ppm}$ ). Fragment ion 284.0204 is a unique fragment when compared with lansoprazole, DI-I and DI-III; its elemental composition and molecular structure (refer Table 4 and Figure 7) strongly support to the proposed molecular structure of unknown impurity (DI-II); refer Figure 9.

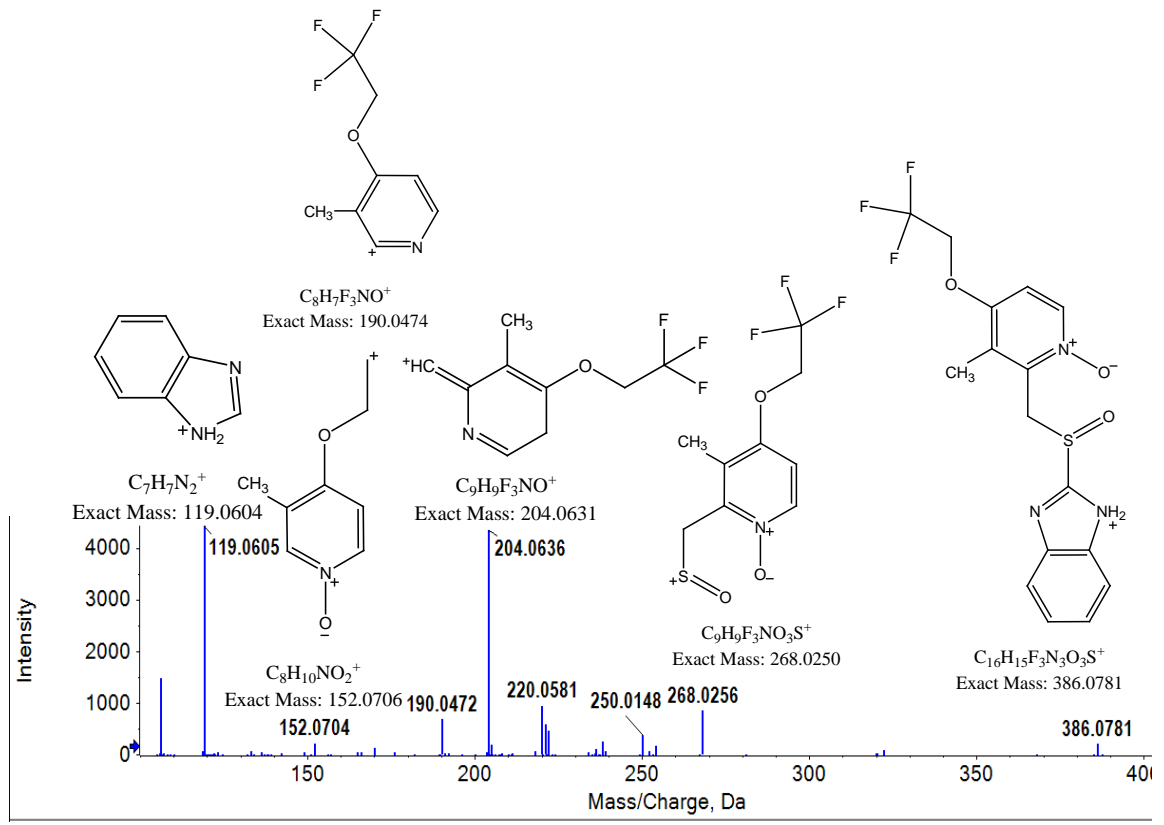

Figure 6. MS/MS spectrum of DI-I, molecular structue of fragment ions along with elemental composition and exact mass. 


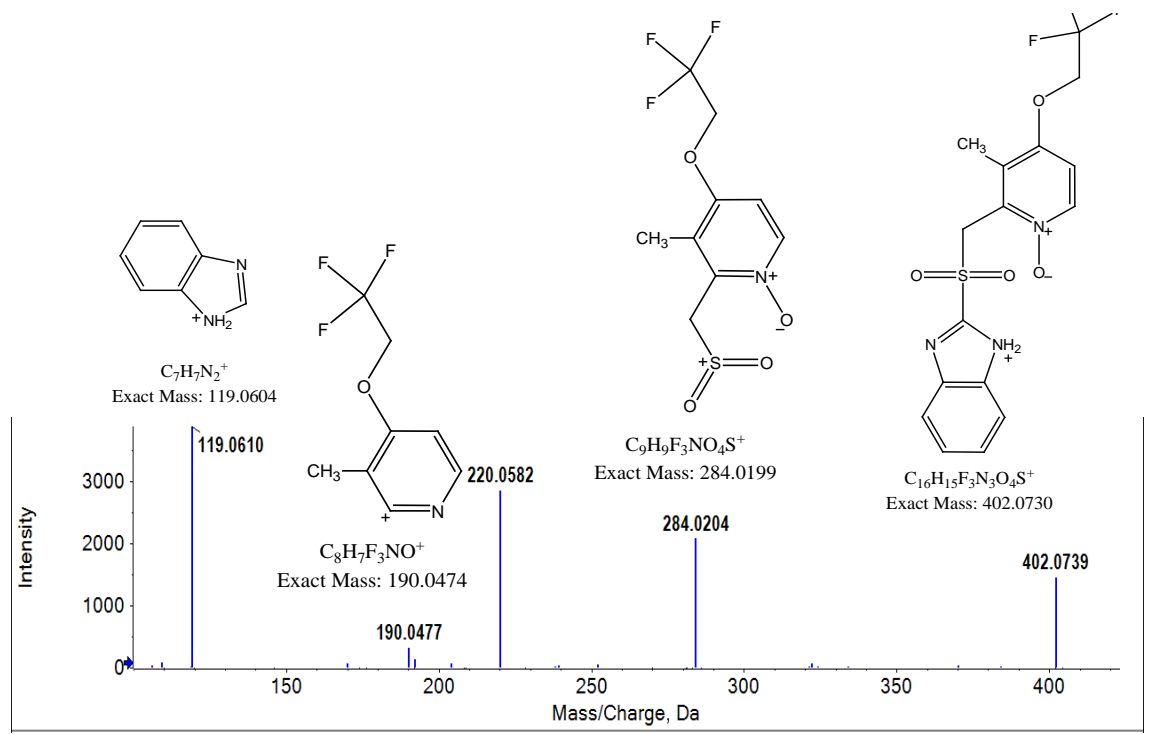

Figure 7. MS/MS spectrum of DI-II, molecular structue of fragment ions along with elemental composition and exact mass.<smiles></smiles>

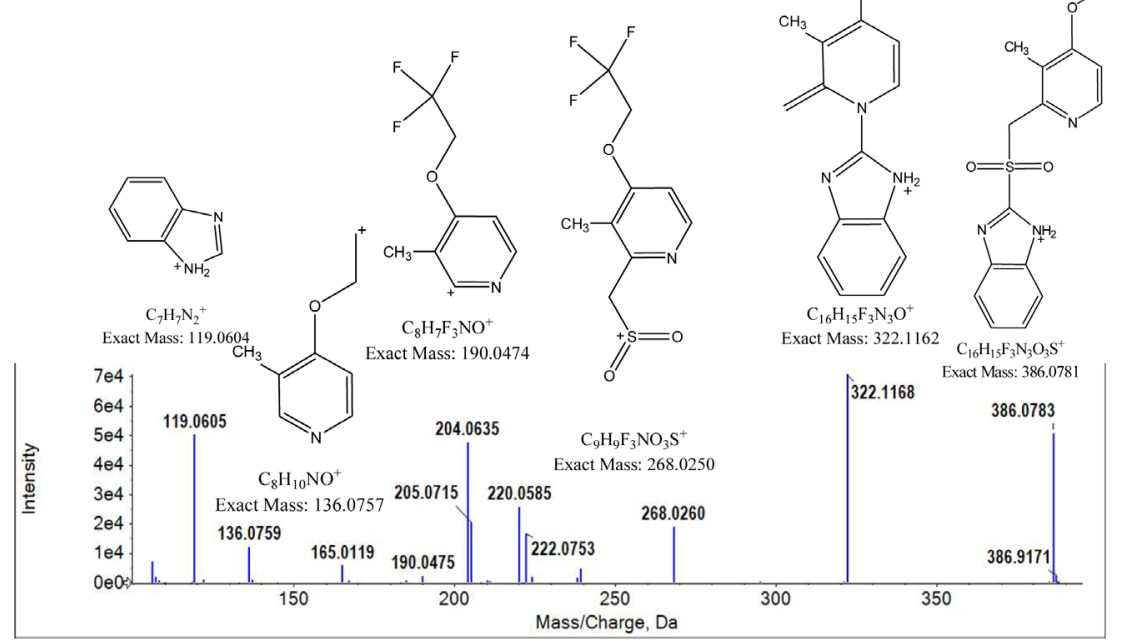

Figure 8. MS/MS spectrum of DI-III, molecular structue of fragment ions along with elemental composition and exact mass.

Full scan spectrum of DI-III exhibit the molecular ion peak as $m / z 386.0785$ $[\mathrm{M}+\mathrm{H}]^{+}$. The TOF MS/MS spectrum of DI-III also exhibited molecular ion $\mathrm{m} / \mathrm{z}$ 386.0783 as $[\mathrm{M}+\mathrm{H}]^{+}$(calculated formula $\mathrm{C}_{16} \mathrm{H}_{15} \mathrm{~F}_{3} \mathrm{~N}_{3} \mathrm{O}_{3} \mathrm{~S}^{+}$, exact mass 386.0781, mass error $0.5 \mathrm{ppm}$ ), molecular ion further fragmented into nine major fragments, the $\mathrm{m} / \mathrm{z} 322.1168,268.0260,222.0753,220.0585,205.0715,204.0635$, $165.0119,136.0759$ and 119.0605. The proposed interpretation of fragments summarized in Table 5 and Figure 8; fragment ion 322.1168 (calculated formula 

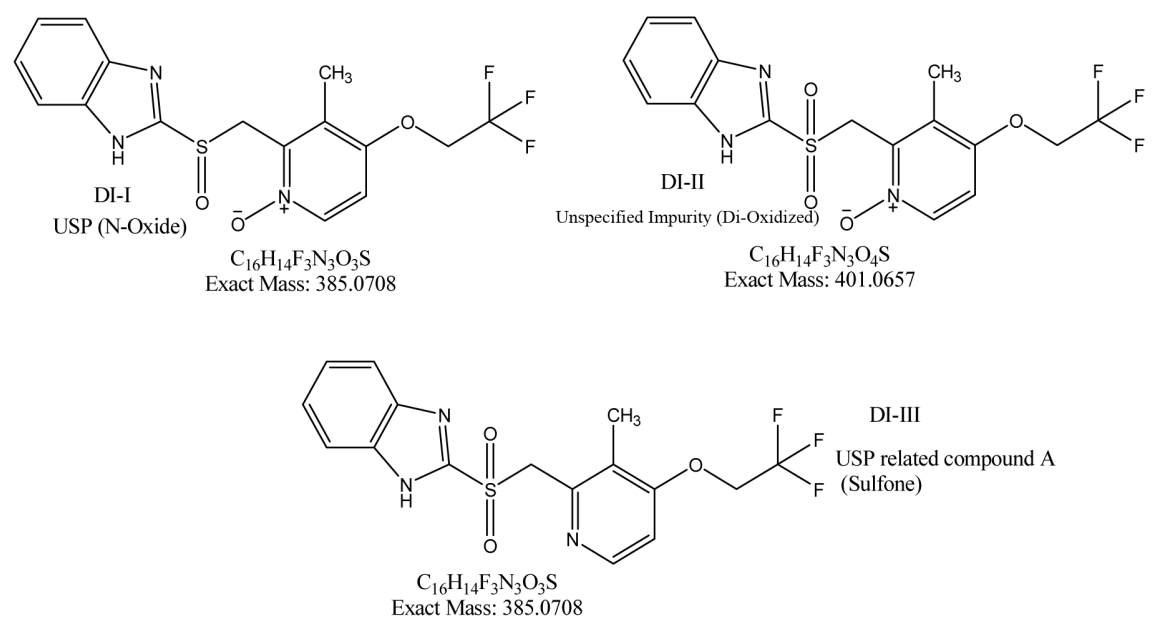

Figure 9. Molecular structure and exact mass of DI-I, DI-II and DI-III.

$\mathrm{C}_{16} \mathrm{H}_{15} \mathrm{~F}_{3} \mathrm{~N}_{3} \mathrm{O}^{+}$, exact mass 322.1162 , mass error 1.9 ppm), fragment ion 268.0260 (calculated formula $\mathrm{C}_{9} \mathrm{H}_{9} \mathrm{~F}_{3} \mathrm{NO}_{3} \mathrm{~S}^{+}$, exact mass 268.0250 , mass error $3.7 \mathrm{ppm}$ ), fragment ion 222.0753 (calculated formula $\mathrm{C}_{9} \mathrm{H}_{11} \mathrm{~F}_{3} \mathrm{NO}_{2}^{+}$, exact mass 222.0736, mass error $7.7 \mathrm{ppm}$ ), fragment ion 220.0585 (calculated for $\mathrm{C}_{9} \mathrm{H}_{9} \mathrm{~F}_{3} \mathrm{NO}_{2}^{+}$, exact mass 220.0580, mass error $2.3 \mathrm{ppm}$ ), fragment ion 205.0715, (calculated formula $\mathrm{C}_{9} \mathrm{H}_{10} \mathrm{~F}_{3} \mathrm{NO}^{*+}$, exact mass 205.0709, mass error $2.9 \mathrm{ppm}$ ), fragment ion 204.0635 (calculated formula $\mathrm{C}_{9} \mathrm{H}_{9} \mathrm{~F}_{3} \mathrm{NO}^{+}$, exact mass 204.0631, mass error $2.0 \mathrm{ppm}$ ), 190.0475, (calculated formula $\mathrm{C}_{8} \mathrm{H}_{7} \mathrm{~F}_{3} \mathrm{NO}^{+}$, exact mass 190.0474, mass error 0.5 ppm) and 119.0605 (calculated formula $\mathrm{C}_{7} \mathrm{H}_{7} \mathrm{~N}_{2}^{+}$, exact mass 119.0604, mass error $0.8 \mathrm{ppm}$ ). Fragment ion 268.0260 is common in DI-I and DI-III but the presence of 322.1168 and absence 152.0704 confirms DI-III as USP related compound A.

Molecular structure and exact mass of DI, DI-II and DI-III presented in Figure 9.

\section{Conclusion}

In this study, generic IDA method developed using high-resolution mass spectrometry system to identify and confirm the lansoprazole degradation impurities under oxidative stressed condition. Generic information dependent acquisition (IDA) method with unique dynamic background subtraction (DBS) shown the capabilities to identify and offer high number of relevant fragment ions or $m / z$ values, for the degradation impurities masses at chromatography run; even for the less resolved peaks. Three oxidative degradation impurities $\mathrm{m} / \mathrm{z} 386.0781$ (DI-I), 402.0734 (DI-II) and 386.0785 (DI-III) were identified and confirmed by rational interpretation of HR-MS and HR-MS/MS spectral data. All proposed molecular structures were strongly supported by accurate mass, elemental composition and low mass error. The workflow [19] applied for mass spectral data interpretation, was found efficient and can be applied for identification of unknown impurities and impurities structure verification studies of other small 
organic molecules.

\section{Acknowledgements}

This paper is part of Ph.D. thesis of Dev Kant Shandilya. Author expresses his gratitude to the Dean, Department of Research, Bhagwant University, Ajmer, Rajasthan, India for extending his constant support.

\section{References}

[1] Smith, R.J. and Webb, M.L. (2007) Analysis of Drug Impurities. Blackwell Publishing, Hoboken. https://doi.org/10.1002/9780470988749

[2] ICH Expert Working Group (2006) Impurities in New Drug Substance ICH Q3A (R2) Current Step 4 Version.

[3] ICH Expert Working Group (2006) Impurities in New Drug Products ICH Q3B (R2) Current Step 4 Version.

[4] ICH Expert Working Group (2003) Stability Testing of New Drug Substances and Drug Products ICH Q1A (R2) Current Step 4 Version.

[5] Willoughby, R.C., Sheehan, S.E.D. and Mitrovich, S. (2002) A Global View of LC/MS: How to Solve Your Most Challenging Analytical Problems. 2nd Edition, Global View Publishing, Pittsburgh.

[6] Pramanik, B.N., Bartner, P.L. and Chen, G. (1999) The Role of Mass Spectrometry in the Drug Discovery Process. Current Opinion in Drug Discovery \& Development, 2, 401-417.

[7] Andrews, G.L., Simons, B.L., Young, J.B., Hawkridge, A.M. and Muddiman, D.C. (2011) Performance of a New Hybrid Quadrupole Time-of-Flight Tandem Mass Spectrometer (Triple TOF 5600). Analytical Chemistry, 83, 5442-5446. https://doi.org/10.1021/ac200812d

[8] Kumar, A., Darekar, G., Ramagiri, S., Bhasin, N., Pillai, M. and Shandilya, D.K. (2015) Generic Workflow Using Advanced Analysis and Data Interpretation Tools for Identification of Irbesartan Degradation Products by Liquid Chromatography to High Resolution Mass Spectrometry. ACAIJ, 15, 352-363.

[9] Pillai, M.G., Kumar, A., Sharma, R. and Bhasin, N. (2014) LC-MS Based Workflows for Qualitative and Quantitative Analysis for Homeopathic Preparation of Hydrastis Canadensis. Chromatographia, 77, 119-131. https://doi.org/10.1007/s10337-013-2577-5

[10] Modhave, D.T., Handa, T., Shah, R.P. and Singh, S. (2011) Stress Degradation Studies on Lornoxicam Using LC, LC-MS/TOF and LC-MSn. Journal of Pharmaceutical and Biomedical Analysis, 56, 538-545. https://doi.org/10.1016/j.jpba.2011.06.012

[11] Ragu, B., Ramesh, M., Srinivas, R., Satyanarayana Raju, S. and Venkateswarlu, Y. (2011) Identification and Characterization of Stressed Degradation Products of Prulifloxacin Using LC-ESI-MS/Q-TOF, MSn Experiments: Development of a Validated Specific Stability-Indicating LC-MS Method. Journal of Pharmaceutical and Biomedical Analysis, 56, 560-568. https://doi.org/10.1016/j.jpba.2011.06.021

[12] Wikipedia (2017) Lansoprazole. https://en.wikipedia.org/wiki/Lansoprazole

[13] Lansoprazole. https://www.drugs.com/lansoprazole.html

[14] http://www.rxlist.com/prevacid-drug.htm

[15] USP Monograph. Lansoprazole, 3498, USP 37. USP.

[16] Rao, P.V. and Kumar, M.N. (2013) A Novel, Validated Stability-Indicating UPLC 
Method for the Estimation of Lansoprazole and Its Impurities in Bulk Drug and Pharmaceutical Dosage Forms. Scientia Pharmaceutica, 81, 183-193. https://doi.org/10.3797/scipharm.1210-09

[17] Battu, S. and Pottabathini, V. (2015) Hydrolytic Degradation Study of Lansoprazole, Identification, Isolation and Characterization of Base Degradation Product. American Journal of Analytical Chemistry, 6, 145-155. https://doi.org/10.4236/ajac.2015.62013

[18] Luo, Y., Xu, Li., Xu, M., Feng, J. and Tang X. (2012) A Validated, Specific, Stability-Indicating HPLC Method for Determination of Lansoprazole Enteric Capsules and Related Impurities. Asian Journal of Pharmaceutical Sciences, 7, 149-154.

[19] Shandilya, D.K., Joseph, P.E. and Kantamreddi, V.S.S. (2017) Interpretation of Full Scan Atmospheric Pressure Ionization Mass Spectra (MS) and Collision Induced Dissociation Fragmentation Spectra (MS/MS) of Small Organic Molecules-A Mini Review. Systematic Reviews in Pharmacy, 8, 23-25.

https://doi.org/10.5530/srp.2017.1.9

[20] Shandilya, D.K., Joseph, P.E. and Kantamreddi, V.S.S. (2017) Prediction of the Fragmentation Pathway of Atorvastatin by Using High Resolution Collision Induced Dissociation (HR-MS/MS) Spectral Data. Open Access Library Journal, 4, e3473. https://doi.org/10.4236/oalib.1103473

[21] Blanc Le Yves, J.C. and Bloomfleld, N. Real Time Dynamic Background Subtraction: Improving the Automated Ion Selection Process. Technical Note, Applied Biosystem.

\section{Submit or recommend next manuscript to SCIRP and we will provide best service for you:}

Accepting pre-submission inquiries through Email, Facebook, LinkedIn, Twitter, etc. A wide selection of journals (inclusive of 9 subjects, more than 200 journals) Providing 24-hour high-quality service User-friendly online submission system Fair and swift peer-review system Efficient typesetting and proofreading procedure Display of the result of downloads and visits, as well as the number of cited articles Maximum dissemination of your research work

Submit your manuscript at: http://papersubmission.scirp.org/ Or contactijamsc@scirp.org 